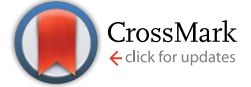

Cite this: RSC Adv., 2017, 7, 12599

Received 15th December 2016 Accepted 12th February 2017

DOI: $10.1039 / c 6 r a 28243 a$

rsc.li/rsc-advances

\section{Preparation, characterization and tribological mechanism of nanofluids}

\begin{abstract}
Linghui Kong, Jianlin Sun* and Yueyue Bao
A nanofluid is a dispersion of nanoparticles in a base fluid and it has been a hot topic of great interest in recent years primarily due to its potential application in various fields. This review presents an overview of the remarkable progress on nanofluids during the past two decades. Nanoparticles have been investigated intensively as an additive for lubricants due to their special tribological properties. This article is focused on various synthetic methods and characterization techniques of nanofluids. Factors enhancing the stability and lubrication mechanism have been delineated in detail. Although nanofluids are potential candidates for tribological applications, there are still many challenges to overcome. These challenges involve the long term stability of nanofluids and validation of lubrication mechanisms. Especially, nanofluid stability and high costs of production are obstacles for the application of nanofluids. The current review also discusses the problems of nanofluids applied in lubrication.
\end{abstract}

\section{Introduction}

Advanced lubricants can improve productivity through energy saving and prolonging the life cycle of machinery. "Nanofluids" was coined by Choi in 1995. Since then, nanofluids have been studied in many fields, including the chemical industry, biomedical applications, electronics cooling, environment engineering and waste heat recovery. And this situation is also true in the tribology. Studies have shown that the dispersion of small amounts of nano-scale solid particles in base fluids, commonly known as nanofluids, enhanced the anti-wear and

School of Materials Science and Engineering, University of Science and Technology Beijing, Beijing 100083, P. R. China. E-mail: sjl@ustb.edu.cn; Tel: +861062333768 friction reduction properties. $\mathrm{Xu}^{1}$ prepared $\mathrm{MoS}_{2}$ hollow spheres with an average diameter of $70 \mathrm{~nm}$ by addition of $\mathrm{TiO}_{2}$ and studied its tribological properties in rapeseed oil. The result revealed that the wear was significantly alleviated by the produced lubricating film containing $\mathrm{MoS}_{2} / \mathrm{TiO}_{2}$ nanoparticles. $\mathrm{Ma}^{2}$ investigated the anti-wear and friction performance of $\mathrm{KH}$ 560 modified $\mathrm{ZrO}_{2}$ in machine oil, and found that the anti-wear and friction reduction abilities of the oil with added $\mathrm{ZrO}_{2}$ nanoparticles were considerably improved. $\mathrm{Liu}^{3}$ reported that surface-modified mixed rare earth nanoparticles with a size less than $30 \mathrm{~nm}$ as the additive exhibit excellent anti-wear, load carrying and good friction reduction capacities in base stock.

Currently, dispersing nanoparticles in lubricating oils is still a challenge for application of nano-additives. ${ }^{4}$ Stability of

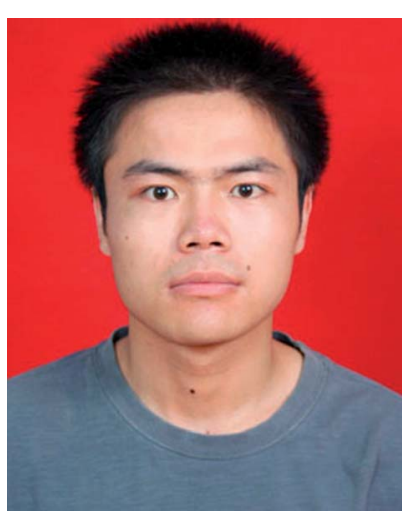

Mr Linghui Kong (born in China in 1987) obtained his master degree from Zhengzhou University, China, in 2012, and then continued his studies for PhD degree in the University of Science and Technology, Beijing, China from the year of 2014. He specializes in dispersion of nanoparticles in aqueous lubricated oils and lubrication mechanism of nanoparticles during different contact conditions. He is currently the coauthor of 4 papers and 2 patents.

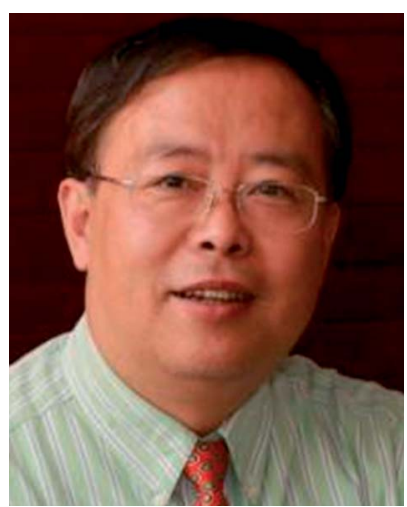

Dr Jianlin Sun (born in China in 1963) obtained bachelor degree from National Defense University of Technology in 1985, and continued his studies for master and doctoral degree PhD in the Central South University of Technology, Changsha, China. He is currently a professor and researcher in the University of Science and Technology, Beijing, China. He specializes in Lubrication Mechanism in MetalForming, Evaluating of Lubricating Performances and the Rolled Surface Quality and nano lubrication as well. Also he is co-author of 3 books, 128 papers and 20 patents. 


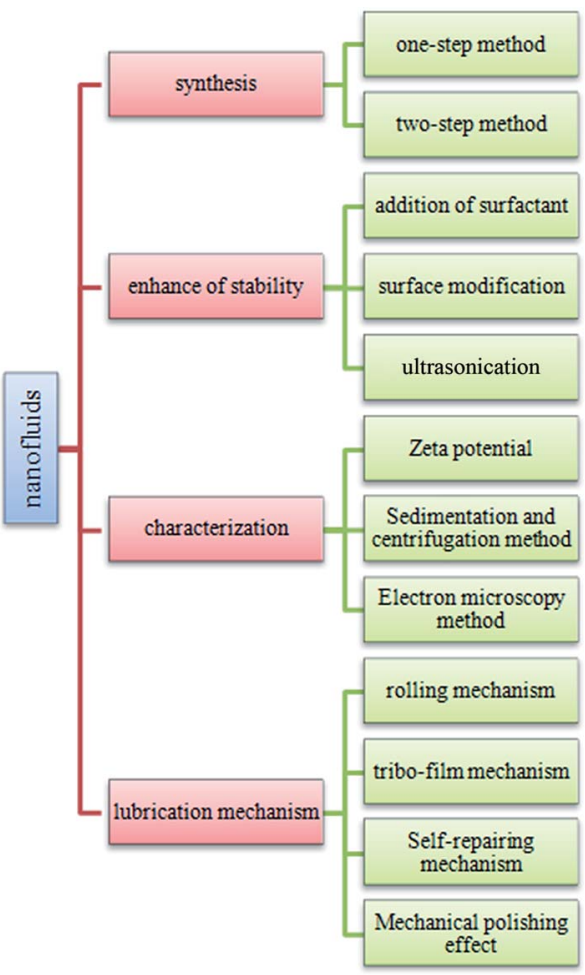

Fig. 1 Structure of the current review.

nanofluids depends on their preparation methods. There are various disagreements in the reported paper of nanofluids stability. The main factor affecting the stability of nanofluid is the tendency of nanoparticles towards aggregation due to the strong van der Waals attractive force. To overcome this obstacle, a series of solutions have been put into practice, such as addition of dispersant,,$^{5-7}$ surface modification of nanoparticles ${ }^{8}$ and ultrasonic dispersion. ${ }^{9}$

When nanoparticles are used as additives of lubricating oils, four lubrication mechanisms may be available: rolling of nanospheres; ${ }^{\mathbf{1 0} 11}$ self-repairing effect because of the minimal size; ${ }^{12}$ tribofilm formation as results of tribochemical reaction; ${ }^{13-18}$ polishing effect. ${ }^{19,20}$

This paper aims at focusing on the existing knowledge and research gap in all fields of nanofluids from synthesis of

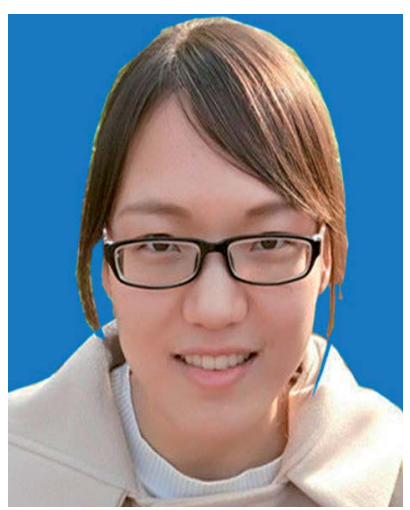

M. S. Yueyue Bao (born in China 1992) obtained bachelor degree from Hohai University in 2015, and continued her master degree in University of Science and Technology Beijing, China. She majored in lubrication mechanism of nano-SiO ${ }_{2}$ in metalforming and effect in surface qualities of metal. nanofluids and to their research in tribological field. An exclusive review section on characterization techniques, improved and recent theoretical models for lubricating developed by different researchers are presented in this review paper as well. The structure of this review is given in Fig. 1.

\section{Preparation of nanofluid}

Nanoparticles, as the additives for nanofluids, play an important role in improving anti-wear, friction reduction and load carrying capacities of nanofluids. Various types of nanoparticles, such as metallic nanoparticles, metallic oxide, have been studied in recent years. Two preparation methods of nanofluids have been reported.

\subsection{Single-step method}

Single-step method is a procedure that combines the production of nanoparticles with the synthesis of nanofluids. Vapour Deposition, developed by Choi in $2001,{ }^{21}$ is the most commonly used single-step method at present. The principle is shown in Fig. 2. Under the centrifugal force of the rotating disk, a flowing thin film of base liquid is formed on the wall of the vessel. Raw material is heated and evaporated in a resistively heated crucible, the vapor is condensed into nano-sized particles when it contacts the cold base liquid film, and nanofluid is obtained. Akoh et al. ${ }^{22}$ developed another single-step direct evaporation method, which is similar to Vacuum Evaporation onto a Running Oil Substrate (VEROS). Extremely fine particles with the average size of about $0.25 \mathrm{~nm}$ were obtained with this method. To successfully prepare well dispersed $\mathrm{Cu}$ nanofluid, a modified VEROS method was proposed by Eastman. ${ }^{23}$ In this method, $\mathrm{Cu}$ particles were produced by direct evaporation into a low vapor pressure liquid. Zhu et al. ${ }^{24}$ presented a single-step chemical process for the preparation of $\mathrm{Cu}$ nanofluid, in which $\mathrm{CuSO}_{4} \cdot 5 \mathrm{H}_{2} \mathrm{O}$ is partly reduced with $\mathrm{NaH}_{2} \mathrm{PO}_{2} \cdot \mathrm{H}_{2} \mathrm{O}$ in ethylene glycol under microwave irradiation. The TEM micrograph reveals that the size of most $\mathrm{Cu}$ particles is in the range of $10 \mathrm{~nm}$ and $20 \mathrm{~nm}$. Choi et al. ${ }^{25}$ also developed a Direct Evaporation Condensation method. The main advantage of this method is the good controllability of nanoparticle size and long term stability of nanofluid. Lo et al. ${ }^{26}$ introduced a novel system was to prepare CuO nanofluid. The method is based on Submerged

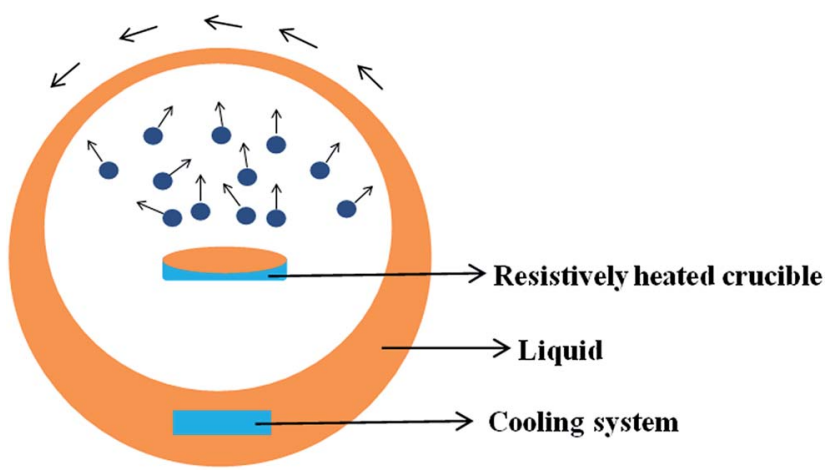

Fig. 2 Schematic diagram of Vapour Deposition method. 
Arc Nanoparticle Synthesis System (SANSS) technique. SANSS mainly consists of a heating source, a state control system and a cooling unit.

Solubility of nanoparticles is relevant to their size, the relationship can be described by eqn (1)

$$
\frac{R T}{M} \ln \frac{s_{1}}{s_{2}}=\frac{2 \sigma}{\rho}\left(\frac{1}{a_{2}}-\frac{1}{a_{1}}\right)
$$

where $s_{1}, s_{2}, a_{1}, a_{2}$ are separately the solubility, size of the two kinds of nanoparticles. If there is wide size distribution of nanoparticles in the base fluid, according to eqn (1), the nanoparticles of small size will diffuse toward big size particles and deposit on the surface of the big particles, age of colloid is formed in this way. Thus nonuniform size distribution is the most important factor that contributed to aggregation of nanoparticles in the base fluid. There is a narrow size distribution of nanofluids that prepared by single step method since the nanomaterial is directly condensed into nano-sized particles from the vapor state, thus less aggregation is involved.

Two main advantages were involved by using single-step method. The first advantage is no drying, storage or dispersing process is needed, thus reducing cost of production. The other advantage is low aggregation of nanoparticles predominately due to the first advantage mentioned above, it is extremely important to ensure the long term stability of nanofluids. The major disadvantage of single-step method is that it is merely compatible with low vapor pressure base fluids.

\subsection{Two-step method}

The sketch of two-step method is shown in Fig. 3. Currently, two-step method is the most commonly used method for the preparation of nanofluids. Generally, two procedures are involved in this method. The first procedure is the synthesis of nanomaterial, which is usually in the form of dry powder. The second procedure is the dispersion of nanomaterial in the base liquid such as water, ethanol and ethylene glycol. During this procedure, some measures, for instance, addition of dispersant or sonication, are generally carried out to enhance the stability of the resulting nanofluids.

Several nanofluids prepared with two-step method are listed in Table 1. Peng ${ }^{27}$ successfully prepared AlN/ethanol nanofluids with the aid of ultrasonic homogenizer. The preparation process was approximately as follows: firstly mixed the AlN and castor oil in the ethanol with vigorous stirring, then placed the mixture in an ultrasonic homogenizer for 10 minutes and eventually well dispersing AlN/ethanol nanofluid was gained.

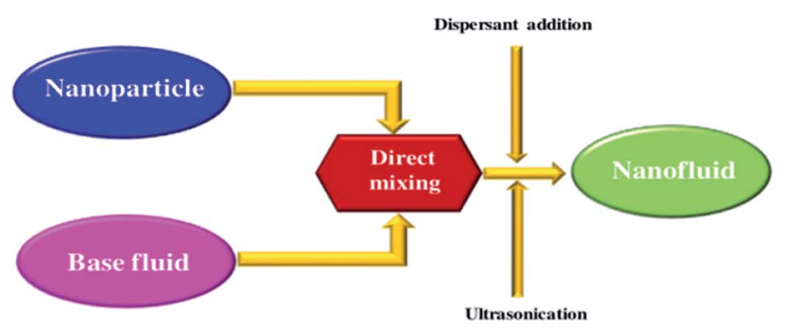

Fig. 3 Preparation process of nanofluids by two-step method. ${ }^{59}$
Table 1 Summary of nanofluids prepared by two step method

\begin{tabular}{lll}
\hline Nanoparticles & Base fluids & Ref. \\
\hline $\mathrm{TiO}_{2}$ & Oil, bidistilled water, deionized water, EG-TNT & $28-31$ \\
$\mathrm{ZnO}$ & PEG, EG, deionized water & $32-34$ \\
$\mathrm{SiO}_{2}$ & TH66, deionized water, methanol & $35-37$ \\
$\mathrm{CuO}$ & Oil, deionized water, oleic acid & $38-40$ \\
$\mathrm{Al}_{2} \mathrm{O}_{3}$ & CMC, EG, deionized water, WEG50 & $41-44$ \\
$\mathrm{Fe}_{3} \mathrm{O}_{4}$ & Kerosene, EG/water & 45,46 \\
$\mathrm{Cu}$ & R113, EG, deionized water, transformer oil & $47-50$ \\
$\mathrm{Ag}$ & Deionized water & 51 \\
$\mathrm{CNTs}$ & Ammonia, deionized water, glycol, EG & $52-55$
\end{tabular}

Diathermic oil based $\mathrm{TiO}_{2}$ nanofluid was fabricated via two-step method, where oleic acid (OA) was used as the dispersant to ensure the stability of nanofluid. ${ }^{28}$ Xuan et al. ${ }^{50}$ prepared $\mathrm{Cu}$ nanofluids of both water and transformer oil, well dispersed nanofluids were achieved when the percentage of dispersant amount reached $22 \mathrm{wt} \%$ of $\mathrm{Cu}$ nanoparticles. Two-step method was also used for the synthesis of CNT based nanofluids. Numerous of CNT nanofluids were prepared by dispersion of CNTs in various base fluids. ${ }^{52-55}$

Compared with single-step method, the main disadvantage of two-step method is the instability of nanofluids because of the high surface energy of nanoparticles. ${ }^{56}$ Besides, the process of drying, storage and transportation of nanoparticles was inevitable in twostep method. However, this method also has advantages. Twostep method enables making nanofluids at a very large scale. ${ }^{57}$ Moreover, it could be used to make almost all kinds of nanofluids. ${ }^{58}$

\section{Stability mechanisms of nanofluids}

Stability is the most crucial issue for nanofluids because of the tendency of mutual attraction between nanoparticles which caused by high surface energy of nanoparticles. According to DLVO theory, there are two forces between nanoparticles, the one is van der Waals attractive force between nanoparticles while the other is electrical double layer repulsive force. The attractive potential energy is given by Hamaker formula, which is listed as eqn (2)

$$
V_{\mathrm{A}}=-\frac{A r}{12 H}
$$

where $V_{\mathrm{A}}$ is the attractive potential energy; $A$ is the Hamaker constant. If there is no direct contact of electrical double layer between two particles, repulsive potential energy is given by eqn (3).

$$
V_{\mathrm{R}}=\frac{64 \pi r n_{0} k T \gamma_{0}^{2}}{\kappa^{2}} \exp (-\kappa H)
$$

The stability of nanofluids is the result of the two opposing forces.$^{60}$ If the repulsive force is much larger than the attractive force, and can overcome the attraction during the collision process due to Brownian movement, the nanofluid is in a relative stable state. Otherwise, the nanofluid is in an unstable state. Steric repulsion and electrostatic repulsion are the two types of mechanisms through which nanofluids are stabilized, as shown in Fig. 4. According to steric repulsion, 


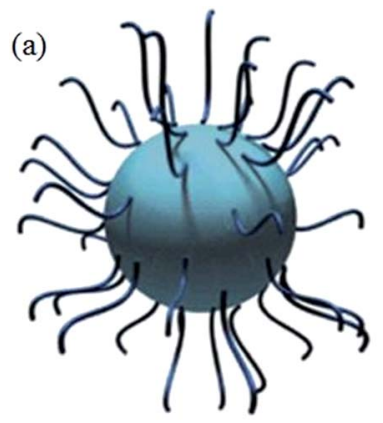

(b)

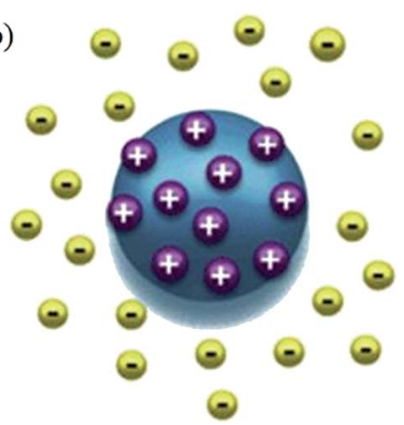

Fig. 4 Type of nanofluids stabilization (a) steric stabilization (b) electrostatic stabilization. ${ }^{61}$

several surfactants, such as SDBS, SDS, CTAB, can prevent the aggregation of dispersed nanoparticles in nanofluids. These surfactants usually have two tails, one is hydrophilic and the other is hydrophobic. The hydrophilic tail adsorbs onto the surface of nanoparticles with a long loop and the hydrophobic tail extends out into the nanofluids. Thus, steric stabilized nanofluids remain well dispersed and sustain for a long period. In electrostatic stabilization, the nanoparticles in nanofluids bear some charge because of adsorption of ions. Electrical double layer is created around nanoparticles, and the repulsive force produced by electrical layer will offset the attractive force between nanoparticles.

Avraham $^{62}$ investigated the role of carbon chain in 3-alkyl thiophenes on the dispersing of CNTs in the organic solvents, and concluded that the stability was not only relied on the stacking of the polymer backbone onto the CNTs, but also on the steric repulsion of carbon chain of adsorbed 3-alkyl thiophenes. Yang et al. ${ }^{63}$ prepared $\mathrm{Al}_{2} \mathrm{O}_{3}$ ammonia/water nanofluids with sodium dodecyl benzene sulfonate (SDBS) as the dispersant. The stability of the nanofluids with different amount of SDBS was studied with light absorbency ratio index method. The results shows that with the increasing amount of surfactant, the stability of nanofluid is lowered first, and then improved, and then is lowered again. In this study, the stability of the nanofluid was first calculated based on the simplified dispersion model and the calculation agreed with the experimental results.

\section{Techniques of nanoparticles dispersed in nanofluids}

Some chemical and physical techniques can be used to modify the surface of nanoparticles to obtain a stable nanofluid through two-step method. Addition of surfactants and surface treatment of nanoparticles are discussed in the follow section. Sonication is discussed as well.

\subsection{Addition of dispersant}

Addition of dispersant is an effective method to prevent the aggregation of nanoparticles, as the dispersant acts as a bridge between nanoparticles and base fluids, creating continuity between nanoparticles and base fluids. ${ }^{64}$ Addition of dispersants lowers the surface tension of base fluids and improves the immersion of nanoparticles.

Commonly used dispersants are listed in Table 2. $\mathrm{Gu}^{65}$ prepared $\mathrm{OA} / \mathrm{La}-\mathrm{TiO}_{2}$ via sol-gel method in association with surface modification with oleic acid (OA). The $\mathrm{OA} / \mathrm{La}-\mathrm{TiO}_{2}$ nanoparticles were spherical with an average size of about $20 \mathrm{~nm}$. $\mathrm{Zin}^{66}$ studied the stability of nano $\mathrm{Cu}$ in ethylene glycol, where SDS or PVP were used as dispersant. PVP proved to be a better stabilizer. Xia et al. ${ }^{67}$ compared the effects of PVP and SDS on the stability of $\mathrm{Al}_{2} \mathrm{O}_{3}$ nanofluids and found that both PVP and SDS can provide excellent stabilization for $\mathrm{Al}_{2} \mathrm{O}_{3}$ nanofluids. Gao et al. ${ }^{68}$ synthesized $\mathrm{TiO}_{2}$ nanoparticles with the average size of $30 \mathrm{~nm}$, and then prepared $\mathrm{TiO}_{2}$ nanofluid with the aid of oleic acid (OA) as the dispersant. Commercially available $\mathrm{Al}_{2} \mathrm{O}_{3}$ nanofluid was prepared by two-step method, where SDS was used as the surfactant. ${ }^{69} \mathrm{Li}^{70}$ compared the stability of $\mathrm{CuO} /$ water nanofluids with and without CTAB dispersant. The results revealed that CTAB could stabilize $\mathrm{CuO}$ nanofluid for one week, while in the nanofluid without CTAB aggregation occurred quickly. The stability of CNTs nanofluids were also enhanced in the presence of SDBS, Triton X-100, or gum arabic. ${ }^{71-74}$

Addition of dispersant is an effective and economic method to enhance the stability of nanofluids. However, the presence of dispersant can increase the viscosity of nanofluids. Generally, the tribological property of lubricant oil is enhanced with the increase of its viscosity. However, it is not the case for lubricant containing nanoparticles. High viscosity of nanofluids is bound to affect the liquidity of nanoparticles in the base fluid. As a result, insufficient number of nanoparticles enters the tribo-pairs when superfluous dispersant is added, and ultimately deteriorate the

Table 2 Widely used dispersants and their structure

Dispersants Structure formula

Polyvinyl pyrrolidone (PVP)

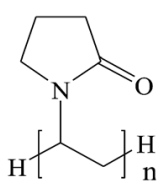

Sodium dodecyl sulphate (SDS)

Oleic acid (OA)<smiles>CCCCCCCCCCCCS(=O)(=O)O</smiles><smiles>CCCCCCCCCCCCCCCCCC(C)C</smiles>

Hexadecyl trimethyl ammonium bromide (CTAB)<smiles>CCCCCCCCCCCCCCCC[N+](C)(C)C</smiles>

Polyacrylic acid sodium (SAAS)

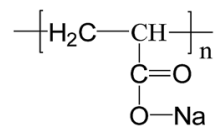

Sodium dodecyl benzene sulfonate (SDBS)

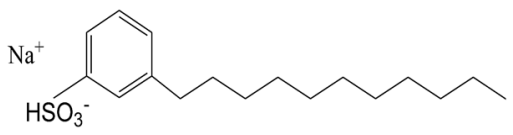


tribological property of lubricant. Additionally, dispersants are usually sensitive to temperature. At high temperature, dispersants could decompose and thus stay out of action.

\subsection{Surface modification of nanoparticles}

The surface of nanoparticles can be modified by functionalization. Simply adding functionalized nanoparticles into the base fluid, long term stability can be achieved. Wang ${ }^{75}$ developed an economic method for surface functionalization of gold nanoparticles, mixed DNA/PEG polymers were instead of original pure DNA in his method, sketch of functionalization was listed in Fig. 5. Compared with pure DNA modification, only a little amount of DNA was used to get same effect. Kim et al. ${ }^{76}$ prepared stable methanol based $\mathrm{CeO}_{2}$ nanofluid by dispersing the surface-modified ceria $\left(\mathrm{CeO}_{2}\right)$ nanoparticles in methanol using ultrasonicator. Alzatecarvajal ${ }^{77}$ attempted modified graphene oxide and nanodiamond by amide functionalization, in this method, a solvent-free gas-phase treatment was involved, aromatic and aliphatic amines were also used. Finally he proved that amidation was the only possible route for functionalization of diamond. Pati et $a .^{78}$ reported a simple approach to produce stable $\mathrm{Fe}_{3} \mathrm{O}_{4}$ nanofluid in the absence of dispersant: $\mathrm{Fe}_{3} \mathrm{O}_{4}$ nanoparticles were first prepared, separated, and washed with acetone and water, followed by soaking in $\mathrm{NaOH}$ solution and drying at $150{ }^{\circ} \mathrm{C}$ for 30 minutes, and finally the dried powder was dispersed in water and sonicated for 30 minutes. CNTs were modified with $\mathrm{HNO}_{3}$ and a mixture of $\mathrm{HNO}_{3} / \mathrm{H}_{2} \mathrm{SO}_{4}$, so that hydrophilic functional groups were introduced onto the surface. The CNTs/water nanofluid was subjected to ultrasonication and good stability was achieved despite of the absence of surfactant. ${ }^{79}$ Likewise, Aravind ${ }^{80}$ purified MWCNT with concentrated acids $\left(\mathrm{H}_{2} \mathrm{SO}_{4}, \mathrm{HNO}_{3}\right.$ and $\mathrm{H}_{2} \mathrm{SO}_{4} / \mathrm{HNO}_{3}$ mixture) to introduce hydrophilic functional groups and satisfactory result was obtained.

\subsection{Ultrasonication}

Ultrasonication is an accepted physical technique to disperse agglomerated nanoparticles into base fluid. However, it is always accompanied with chemical treatment, rather than an independent dispersing method.
Teng ${ }^{81}$ prepared $\mathrm{Al}_{2} \mathrm{O}_{3}$ /water nanofluids with different concentration, the nanofluids were dispersed by cationic dispersant (chitosan) accompanied with ultrasonic treatment. $\mathrm{TiO}_{2}, \mathrm{ZnO}$ and $\mathrm{Al}_{2} \mathrm{O}_{3}$ were dispersed in water/EG mixture fluid, the SDS was used as dispersant, followed by sonicated treatment for 1 hour aimed at enhancing stability of nanofluids. ${ }^{82}$ $\mathrm{CuO} /$ water nanofluid was prepared via sonicated treatment, long term stability was achieved although no surfactant was added. ${ }^{83}$ The surface of the MWCNT is modified by acid treatment and then dispersed in water/ethylene glycol by sonication for nearly $40 \mathrm{~min}$ using a $100 \mathrm{~W}, 40 \mathrm{kHz}$ ultrasonicator. ${ }^{80}$

Although ultrasonication has been accepted as an essential step in the preparation of nanofluids through two-step method, there is no standard for ultrasonic time and wave. And what type pulse mode should be taken is also kept unknown. Mahbubul ${ }^{84}$ investigated the effect of ultrasonication duration on the stability of $\mathrm{Al}_{2} \mathrm{O}_{3}$ /water nanofluids, where the particles size distribution and zeta potential were analysed to explore the dispersion of $\mathrm{Al}_{2} \mathrm{O}_{3}$ in water, and they found that cluster size decreased while zeta potential increased with the increase of ultrasonication duration in the first 3 hours. Nguyen $^{85}$ evaluated the main parameters of ultrasonication such as power and irradiation modes on the cluster size of $\mathrm{Al}_{2} \mathrm{O}_{3}$ in the aqueous solutions, he pointed out that both continuous and pulsed irradiation were helpful to reduce the size of $\mathrm{Al}_{2} \mathrm{O}_{3}$ cluster, besides, optimal break up efficiency could be achieved at a vibration amplitude of $30 \%$.

There are contradictory results among the literatures about the effect of ultrasonication duration on the stability of nanofluids. So more attempts should be take into effect to explore the optimum duration.

\section{Characterization of nanofluids}

Characterization of nanofluids always reveals several important details such as nanoparticle shape, size, distribution and stability of nanoparticles in base fluid. The most commonly used characterization means include zeta potential analysis, sedimentation method, and electron microscopy.

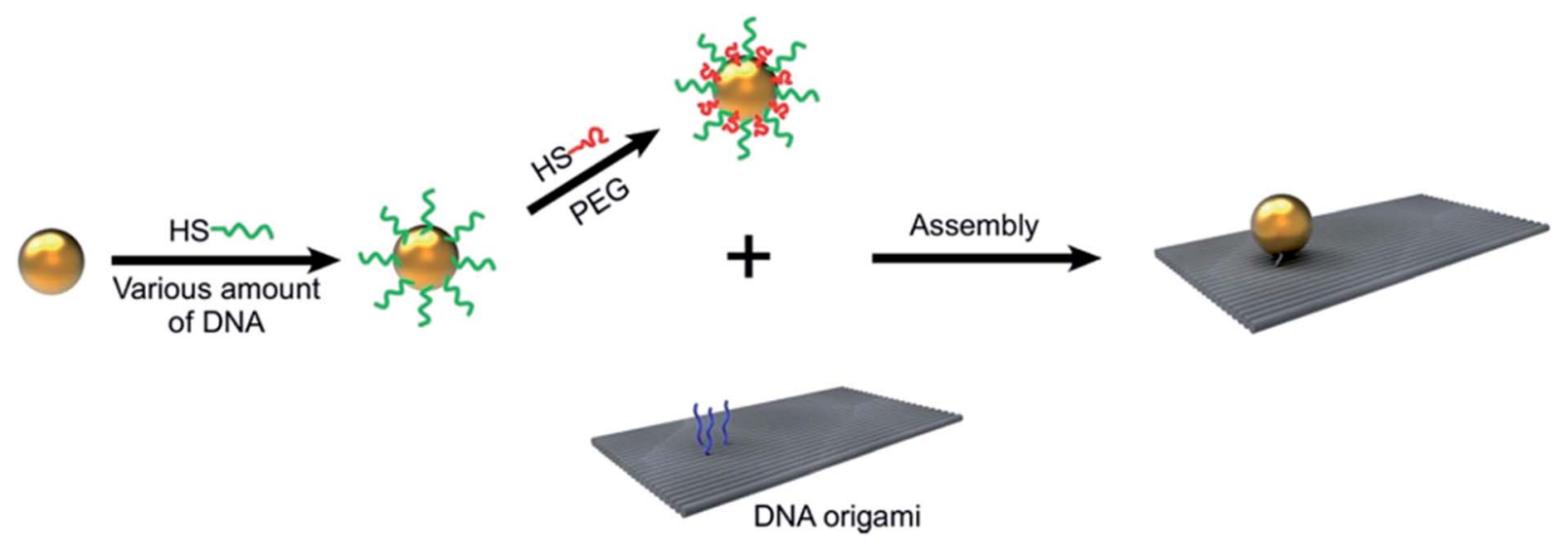

Fig. 5 Surface modification of gold nanoparticles. Reproduced from ref. 75 with permission from the Royal Society of Chemistry. 


\subsection{Zeta potential analysis}

The liquid layer surrounding the particles contains two different regions; an inner region (stern layer) and an outer (diffuse) region. At the inner region ions are firmly bound and at the outer region they are loosely associated. There is a notional boundary inside the outer layer where the particles and ions form a stable entity. The potential at this boundary is known as zeta potential. The main advantage of zeta potential analysis is that it is quick. When the absolute value of zeta potential is high, the repulsive force between nanoparticles will prevail and the nanofluid is in stable state. On the contrary, if the absolute value of zeta potential is low, the attractive force is dominating and the nanofluid tends to aggregate. The relationship is listed in Table 3.

As shown in Table 3, nanofluids with zeta potential higher than $30 \mathrm{mV}$ are relatively stable and above $60 \mathrm{mV}$ show excellent stability with little settling. When zeta potential is below $15 \mathrm{mV}$, a nanofluid is in unstable state, and when zeta potential is closed to $0 \mathrm{mV}$, nanofluid undergoes significant aggregation. Due to a large negative zeta potential in water, $\mathrm{Au} /$ water nanofluids without any dispersant were prepared and exhibit good stability. ${ }^{87} \mathrm{Li}$ et $a .^{88}$ systematically studied the effects of $\mathrm{pH}$, various dispersants, and concentration on zeta potential of $\mathrm{Cu}$ / water nanofluids, and the results showed that at $\mathrm{pH} 9.5$, the absolute value of zeta potential is remarkably higher with the addition of SDBS or CTAB. The aggregation stability of aqueous dispersion of the hydrated anatase and rutile sample in the neutral and alkaline $\mathrm{pH}$ regions were related to the electrostatic stabilization and were explained through DLVO theory. ${ }^{\mathbf{8 9}}$ Stability and zeta potential of graphite nanofluids at different $\mathrm{pH}$ value have been investigated and higher zeta potential was obtained in acidic nanofluid. ${ }^{\mathbf{9 0}}$ Himansu $^{\mathbf{9 1}}$ evaluated the stability of surface modified $\mathrm{CeO}_{2}$ nanoparticles in deionized water by zeta potential method, and found that the value reached $42 \mathrm{mV}$, and $10 \mathrm{mV}$ higher than that of unmodified nanoparticles.

\subsection{Sedimentation and centrifugation}

Sedimentation is the tendency of nanoparticles to settle out of the base fluid in which they are dispersed and finally come to rest against an external force. At steady state, sedimentation velocity of spherical particles is given by Stokes law as eqn (4):

$$
V=\frac{2 g r^{2}\left(\rho-\rho_{1}\right)}{9 \eta}
$$

where $V, R, \rho$ are the sedimentation velocity, radius, and density of nanoparticles, respectively. And $\rho_{1}, \eta$ are density and viscosity of the base fluid, respectively.

Table 3 Relationship between zeta potential and the stability of nanofluids ${ }^{86}$

\begin{tabular}{ll}
\hline Zeta potential $(\mathrm{mV})$ & Stability of nanofluid \\
\hline 0 & Very little or no stability \\
15 & Little stability with settling \\
30 & Moderate stability with sedimentation \\
45 & Good stability with possible settling \\
60 & Excellent stability, little settling
\end{tabular}

According to Stokes law, sedimentation velocity decreases when nanoparticles become smaller. Thus, sedimentation will be slow for smaller nanoparticles relative to large nanoparticles dispersed in base fluid.

Sedimentation and centrifugation are the most commonly used method to investigate the stability of nanofluids because of the simplicity operation. ${ }^{\mathbf{9 2 , 9 3}}$ The stability of nanofluid is directly indicated by the mass or volume of sediment. Nanofluids are considered stable when the concentration or particle size in the supernatant stays constant. $\mathrm{Zhu}^{90}$ evaluated the stability of graphite nanofluids with sedimentation balance method. The results revealed that the nanofluid was more stable when PVP concentration was higher than $0.4 \mathrm{wt} \%$. Angayarkanni and Philip ${ }^{94}$ studied the stability of water based alumina nanofluids at different time intervals by sedimentation and confirmed that the sedimentation rates of different kinds of nanofluids were all in accord with their time depended thermal conductivity measurement.

\subsection{Electron microscopy}

Electron microscopy is generally used to investigate the stability of nanofluids by observing the distribution and aggregation of nanoparticles. Generally it is used for characterizing the morphology of nanoparticles before dispersion. Transmission electron microscopy (TEM) provides high resolution images that can reach approximately $0.1 \mathrm{~nm}$ in case of lattice images. Aggregation of nanoparticles within the nanofluids can be directly monitored with TEM.

$\mathrm{Li}^{95}$ observed the morphology of $\mathrm{Y}_{2} \mathrm{O}_{3}$-stabilized $\mathrm{ZrO}_{2}$ nanoparticles without and with different surfactant, confirmed that the average size of nanoparticles modified by polyether amine D2000 or PVP-10000 was smaller than modified by PEG-4000 or without any surfactant. Yang ${ }^{96}$ successfully prepared oleic acidmodified lanthanum trifluoride-graphene oxide nanohybrids and observed the morphology with TEM. A typical TEM image of graphene oxide (GO) that presents highly transparent films and $\mathrm{LaF}_{3}-\mathrm{GO}$ structure formed by $\mathrm{LaF}_{3}$ nanoparticles on the surface of GO is seen in his experiment. Nanodiamond-agglomerate on $\mathrm{Cu}$ grid with lacey carbon was characterized with TEM $^{97}$ as well.

The characterization of nanoparticles dispersed in base fluid with TEM, the usual practice is placing a drop of as-prepared nanofluids onto a copper grid coated with a carbon film, and then observes the distribution of nanoparticles on copper grid when base fluid is completely evaporated. There is one point should be noted, the evaporation of base fluid always accompanies with aggregation of nanoparticles. As a result, the characterization mean by TEM is only available when less nanoparticles in base fluid. Cryo-EM may be a potential substitute in the future.

\section{Lubricating mechanisms of nanoparticles in nanofluids}

During the past two decades, the application of nanofluids in lubrication has made substantiates progress. Because of the low melting point and high chemical reactivity, nanoparticles may deposit on the microdefects of rubbing surfaces, and play a role 
of "self-repairing" to a certain degree. This is a great potential advantage of nanofluids on lubrication. Furthermore, the thermal conductivity of nanofluids is usually higher than that of their base fluid, which will help to release the heat produced in the friction and maintain the stability of the tribo-pairs. Finally, most nanoparticles are environmentally friendly, as an alternative of traditional additives which commonly containing sulfur and phosphorus, this is very beneficial to the sustainability of the environment and economy. At present, four lubrication mechanisms are commonly accepted: rolling, polishing, self-mending and tribo-film mechanism. Additionally, nanoparticles are also effective in lubrication because of sintering or melt on the tribo-film.

\subsection{Rolling mechanism}

The friction mode is influenced by the well distributed spherical nanoparticles in the nanofluids, because they can switch from sliding friction to rolling friction. And the load carrying capacity is also improved. $\mathrm{Kao}^{98}$ evaluated the role of spherical $\mathrm{TiO}_{2}$ nanoparticles in friction reduction, and found that $\mathrm{TiO}_{2}$ can reduce the worn scar depth by $80.84 \%$, which might be attributed to rolling effect at high temperature. Luo $^{99}$ synthesized $\mathrm{Al}_{2} \mathrm{O}_{3} / \mathrm{TiO}_{2}$ nanocomposites with an average size of $75 \mathrm{~nm}$, and investigated the friction and wear properties of the nanocomposites in lubricating oil. They found that the addition of $\mathrm{Al}_{2} \mathrm{O}_{3} / \mathrm{TiO}_{2}$ enhanced the tribological performance of the lubricating oil, possibly due to wear behavior switching from sliding friction to rolling friction during the process.

Rolling mechanism theory proposes that two factors contribute to the excellent lubrication effect of nanofluids. One is that the nanoparticles are usually spherical, and they may act as "micro-bearing" during the friction; the other is at high temperature and load, the nanoparticles between two rubbing surfaces become flat and form a "sliding system", which ultimately reduces the friction and wear. Mahdi Khadem ${ }^{\mathbf{1 0 0}}$ explored the tribological behavior of nanodiamond on two kinds of rubbing surfaces with different hardness, and claimed that nano-diamond were likely to get embedded into the surface of the soft specimen, rolling effect occurred only when rubbing surfaces own comparatively higher hardness. The embedded and rolling mechanism is illustrated in Fig. 6.

\subsection{Tribo-film mechanism}

Nanoparticles that well dispersed in nanofluids are liable to form a thin film on the metal surface, and this film is dense and low shear, which can separate the rub surface and reduce the friction. In the process of friction, the nanoparticles in the nanofluid tend to migrate to the rubbing surface due to two factors. The one is that the local high temperature because of friction may arouse nanoparticles' undulations in the rubbing surface is fiercer than that in the nanofluids. Random migration is more active as a result of this undulation, and ultimately increase the chance of nanoparticles migrates to the rubbing surface. The other is that the exoelectrons produced during the friction accelerate to strengthening electric-field the on the rubbing surface, the existence of electrical field may cause the gathering of nanoparticles on the rubbing surface.

Tribo-film mechanism is the most prevailing theory to explain the anti-wear and friction reduction behaviour of nanofluids. Most scientists and researchers attributed better lubrication performance of nanofluids to the fact that nanoparticles being deposited on rubbing surfaces and forming a protect film.

$\mathrm{Wu}^{\mathbf{1 0 1}}$ prepared OA modified $\mathrm{ZnO} / \mathrm{PAO}$ nanofluid and studied the wear mechanism of ZnO nanoparticles in PAO, he declared that nanoparticles could easily enter into sliding metal surface due to their small size. These nanoparticles also proved to be easy to adsorb or deposit on the sliding metal surface to form a surface protective film and thus reduce the friction. Zhang $^{102}$ prepared $\mathrm{Cu} / \mathrm{SiO}_{2}$ nanocomposite and evaluated its tribological properties in water-based lubricant, and found that during the friction process, a protective and lubricious film composed of $\mathrm{Cu}$ and a small amount of Fes, $\mathrm{FeSO}_{4}$ and $\mathrm{SiO}_{2}$ is (a)

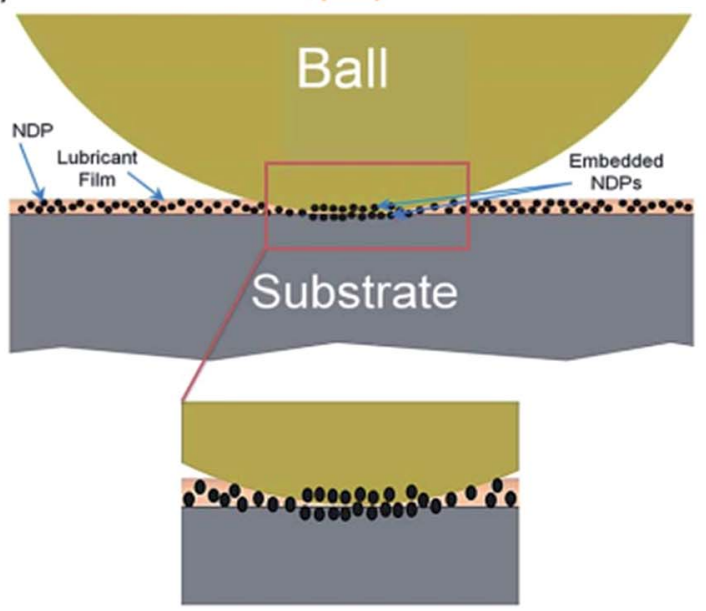

(b)

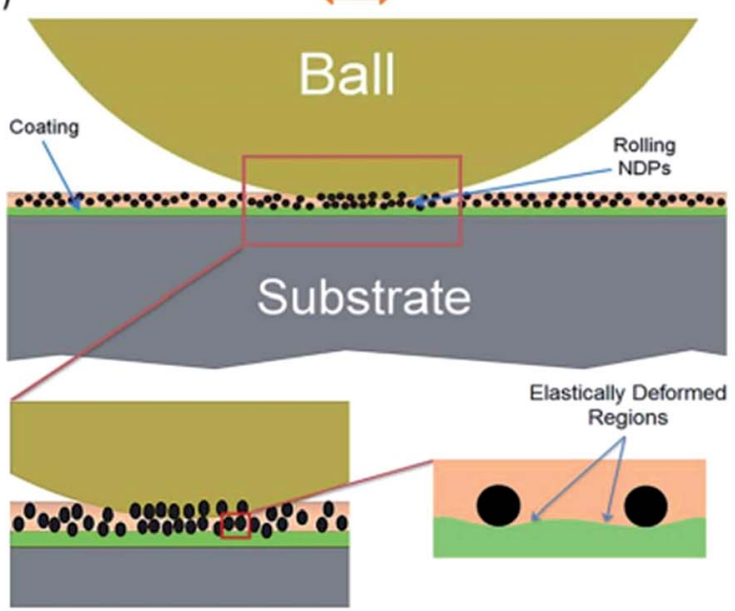

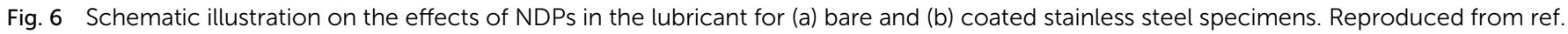
100 with permission from the Royal Society of Chemistry. 
formed on the rubbed steel surface. $\mathrm{Abad}^{103}$ systematically studied the tribological behavior of the Pd NPs dispersed in TBA that carried out by different experimental parameters, the Pt NPs directly deposited onto the contact surface and formed a protective film was been proven in his study. $\mathrm{Chen}^{104}$ synthetized stearic acid-capped cerium borate $\left(\mathrm{SA} / \mathrm{CeBO}_{3}\right)$ and studied the friction and wear performances of $\mathrm{SA} / \mathrm{CeBO}$ in rapeseed oil. The results indicated that $\mathrm{SA} / \mathrm{CeBO}_{3}$ adsorbed and triboreacted to form $\mathrm{B}_{2} \mathrm{O}_{3}, \mathrm{CeO}_{2}$ and $\mathrm{Fe}_{2} \mathrm{O}_{3}$ on the rubbing surface.

Although most scientists claimed that nanoparticles deposited into a protective film on the rubbed surface during the process of friction, as they detected the existence of nanoparticles on the wearing surface. The amounts of deposited nanoparticles were minor or ambiguous. ${ }^{105,106}$ A hypothesis could be proposed. Continuous and dense film might be not formed since a small quantity of deposited nanoparticles were discoverable in these literatures.

The deposition of nanoparticles on a given solid was depended on the surface energy of this solid. Fig. 7 presents the profile sketch of a non-smooth surface, where location A represents asperity while B stands for valley. It's easily concluded that the surface energy of location $A$ is higher than that of location B based on definition of solid surface energy. Thus nanoparticles are more likely to deposit on asperity of solid surface. According to Kong, ${ }^{107}$ heterogeneous deposition of nanoparticles on wearing surface will cause the transition of lubrication mechanism, which is changed from traditional mixed lubrication to a new synergistic lubrication that comprised with asperity, nanoparticles and liquid film, as shown in Fig. 8.

\subsection{Self-repairing mechanism}

Nanoparticles in nanofluids tend to fill the microcracks and repair microdamages via desorption or deposition on rubbing surface, and eventually help to form more flat and smooth surfaces. Usually, a soft metal whose structure is face-centered cubic has the capacity of "self-mending". Wang ${ }^{108}$ pointed out that nano-copper in lubricating oil form a film which separates the friction materials in the friction process. $\mathrm{Ma}^{109}$ studied the effect of $\mathrm{Ag}$ nanoparticles as additives for multi-alkylated cyclopentanes and found that Ag nanoparticles deposited on the friction surfaces to form metallic Ag boundary film. The protective metal film had low shearing stress and contributed to

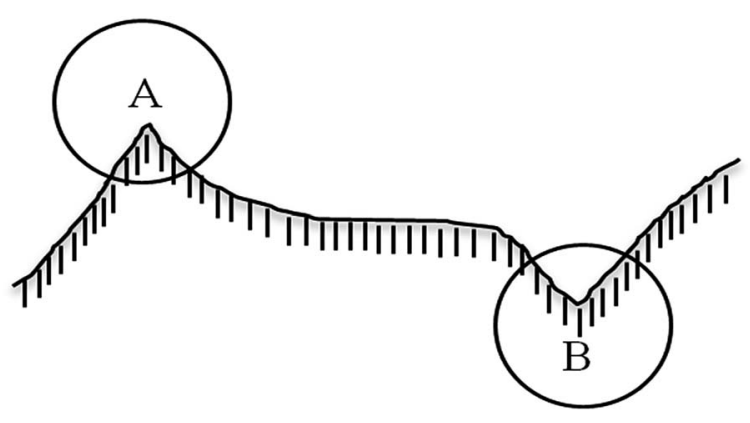

Fig. 7 Profile sketch of a rough solid.

\section{Nanoparticles deposited region}

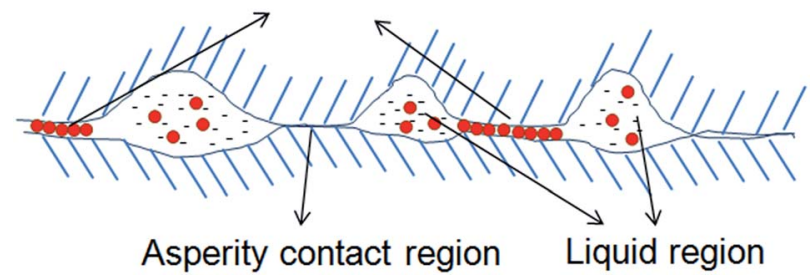

Fig. 8 Sketch of synergistic lubrication model.

preventing the steel-to-steel contact from severe adhesion, scuffing, and seizure. Flores-Castañeda ${ }^{110}$ also concluded that the wear rate reduction by bismuth nanoparticle additives was resulted from the tribo-sintering of nanoparticles on the rubbing surfaces reducing the metal-to-metal contact and acting as a low friction load-bearing film.

It is worth mentioning that, "self-repairing" mechanism is not simply an accumulation of nanoparticles on the rubbing surface. With the decreasing size of nanoparticles, their melting points decline sharply. At the high temperature of rubbing surfaces, these nanoparticles are easily melted or sintered in the microcracks of the contact area, and sequential filler is formed and closely tied to the rubbing surface.

\subsection{Mechanical polishing effect}

Hard nanoparticles in nanofluids act as an excellent polishing tool and play a role in mechanical polishing on rubbing surfaces in the process of friction. The real contacting area of tribo-pairs is increased due to smoother surface polished with hard nanoparticles, which ultimately lowers the friction coefficient and increase the load carrying capacity.

As for the surface with a high roughness, nanoparticles do not exhibit good mechanical polishing effect in terms of improving the tribological properties of the nanofluids, because nanoparticles are too small for the rough surfaces. They can merely repair the surface at the atomic scale. Thus, mechanical polishing effect is more evident when the tribo-pairs have a low surface roughness.

$\mathrm{Chou}^{111}$ observed the surface morphology of 6061 aluminium alloys lubricated separately by commercial oil and nanofluids containing $0.05 \%$ nanodiamond, and found that the surface roughness of aluminium alloy lubricated by nanofluid was decreased by $15 \%$ compared with that lubricated by commercial oil, he pointed that it was polishing action of diamond nanoparticles resulted in the final smoother surface. Tao ${ }^{19}$ evaluated the ball-bearing effect of diamond nanoparticle in base oil, and confirmed that initially the diamond nanoparticles mainly take the effect of surface polishing.

A common phenomenon is that almost all tribological tests prove that the rubbed surfaces lubricated by nano lubricants are much smoother. However, it's not sufficient to attribute it to the polishing effect of nanoparticles, because of self-repairing, nanoparticles are easily deposited filled into the microcracks and grooves and flat surface can also be gained. 


\subsection{Diversification of lubrication mechanism}

In most cases, the nanoparticles do not work by a single lubrication effect, but by two or more mechanisms. Besides, there may be a transition during different mechanisms when changed lubrication condition occurs.

Zareh-Desari ${ }^{\mathbf{1 1 2}}$ studied the lubrication properties of $\mathrm{SiO}_{2}$ and $\mathrm{CuO}$ nanoparticles in vegetable oil, and claimed that two mechanisms were involved in the process of friction, one was the rolling effect and the other was self-repairing mechanism. Zheng $^{113}$ discussed the synergistic lubrication mechanism of $\mathrm{Fe}_{3} \mathrm{O}_{4} / \mathrm{MoS}_{2}$ nanocomposites in aqueous and oil phase media, he argued that nanoball bearing of $\mathrm{Fe}_{3} \mathrm{O}_{4}$ and tribo-film formed with $\mathrm{MoS}_{2}$ might contribute to the friction reducing and antiwear performances of nanofluids.

Although plenty of works have been done on the tribological performance of nanofluids. Most studies in the field of analysis on lubrication mechanism always focus on the effect of nanoparticles. In fact, the mutual interactions among the nanoparticles, dispersant and base fluid should be also taken into consideration.

\section{Problems of nanofluids in lubrication}

Although nanofluids show excellent tribological properties in lubrication, there are still some problems to be solved.

The most important issue is the dispersion and stability of nanoparticles in nanofluids. Nanoparticles have large specific surface area, high chemical reactivity, wide diffusivity and high absorption. All these characteristics are the prerequisite of nanoparticles to form protective film or repair the rubbing surfaces. However, these characteristics also lead to poor stability and dispersion as well. Aggregation of nanoparticles can even cause severe abrasive wear. Usually, surfactants or modification of the surface of nanoparticles can be used to reduce the surface energy of nanoparticles to alleviate the aggregation. However, the selection and appropriate level of surfactants and the improvement of modification technology are still barriers for the application of nanofluids in lubrication.

Another problem in nanofluids is the rough edges related to lubrication mechanisms of nanofluids. The lubrication mechanisms of nanofluids, such as micro-rolling mechanism, still have not been validated by direct experimental data. And some views are somewhat farfetched to explain the lubrication phenomenon. For example, nano-copper shows good tribological properties under high load. Some literatures ${ }^{\mathbf{1 0 8 , 1 1 4 - 1 1 6}}$ tried to explain that by deposition of nano-copper and formation of a protect film. However, copper film itself is not helpful to improve lubrication under high pressure.

\section{Conclusion}

The present review covers the preparation and analytical techniques of nanofluids and the lubrication mechanisms in tribology. Although much progress has been made, it is still a challenge to make a homogenous and long-term stable nanofluid with negligible agglomeration and little effect on the tribological properties.

There are two methods to prepare nanofluids. Nanofluids prepared by single-step method are more stable than those by two-step method. However, the two-step method is applicable in the fabrication of almost all kinds of nanofluids.

Three techniques can be used to improve the stability of nanofluids. Compared to surfactants, surface modification seems to be more effective to achieve stable nanofluids and it exerts less influence on the physicochemical properties of nanofluids. As an auxiliary method, ultrasonication is always accompanied by the addition of surfactant or surface modification.

A common view in several reports is the deposition of nanoparticles in the gaps and grooves of the rubbing surfaces is responsible for improved tribological performance, this claim need to be substantiated with appropriate experimental results and theoretical models. Further detailed tribological studies using DLS, SEM, TEM, AFM and XPS are indispensable for insight into the interaction between nanoparticles and contact surface.

\section{Acknowledgements}

The present study is financially supported by the National Natural Science Foundation of China (51274037).

\section{References}

1 Z. Y. Xu, Y. Xu, K. H. Hu, et al., Tribol. Int., 2015, 81, 139148.

2 S. Ma, S. Zheng, D. Cao, et al., Particuology, 2010, 8(5), 468472.

3 R. Liu, X. Wei, D. Tao, et al., Tribol. Int., 2010, 43(5-6), 10821086.

4 D. Kim and L. A. Archer, Langmuir, 2011, $27(6)$, 3083-3094.

5 V. Zin, F. Agresti, S. Barison, et al., IEEE Trans. Nanotechnol., 2013, 12(5), 751-759.

6 M. H. Kayhani, H. Soltanzadeh, M. M. Heyhat, et al., Int. Commun. Heat Mass Transfer, 2012, 39(3), 456-462.

7 M. Asrul, N. W. M. Zulkifli, H. H. Masjuki, et al., PLoS One, 2013, 68(12), 320-325.

8 T. Luo, X. Wei, H. Zhao, et al., Ceram. Int., 2014, 40(7), 10103-10109.

9 S. M. Alves, B. S. Barros, M. F. Trajano, et al., Tribol. Int., 2013, 65(3), 28-36.

10 F. ChiñAs-Castillo and H. A. Spikes, J. Tribol., 2003, 125(3), 552-557.

11 J. L. Viesca, A. H. Battez, R. González, et al., Tribol. Int., 2011, 44(7), 829-833.

12 G. Liu, X. Li, B. Qin, et al., Tribol. Lett., 2004, 17(4), 961966.

13 X. Zhou, X. Fu, H. Shi, et al., Lubr. Sci., 2007, 19(1), 71-79.

14 A. H. Battez, J. E. F. Rico, A. N. Arias, et al., Wear, 2006, 261(3-4), 256-263.

15 T. Shen, D. Wang, J. Yun, et al., Tribol. Int., 2015, 93.

16 B. Chen, K. Gu, J. Fang, et al., Appl. Surf. Sci., 2015, 353, 326332. 
17 H. L. Yu, Y. Xu and P. J. Shi, Trans. Nonferrous Met. Soc. China, 2008, 18(3), 636-641.

18 F. Zhao, Z. Bai, Y. Fu and D. Zhao, Wear, 2012, 288, 72-77. 19 X. Tao, Z. Jiazheng and X. Kang, J. Phys. D: Appl. Phys., 1996, 29(11), 2932-2937.

20 D. Peng, C. Chen, Y. Kang, et al., Ind. Lubr. Tribol., 2010, 62(2), 111-120.

21 S. U. S. Choi and J. A. Eastman, US Pat. 6221275 B1 [P]. 2001.

22 H. Akoh, Y. Tsukasaki, S. Yatsuya, et al., J. Cryst. Growth, 1978, 45(2), 495-500.

23 J. A. Eastman, U. S. Choi, S. Li, et al., Mater. Res. Soc. Symp. Proc., 1995, 457.

24 H. T. Zhu, Y. S. Lin and Y. S. Yin, J. Colloid Interface Sci., 2004, 277(1), 100-103.

25 U. S. Choi, W. Yu, J. R. Hull, et al., SAE [Tech. Pap.], 2002, 3843.

26 C. H. Lo, T. T. Tsung, L. C. Chen, et al., J. Nanopart. Res., 2005, 7(2-3), 313-320.

27 H. Peng, W. L. Shan, F. Yu, et al., Int. J. Thermophys., 2008, 29(6), 1968-1973.

28 B. Wei, C. Zou and X. Li, Int. J. Heat Mass Transfer, 2017, 104, 537-543.

29 S. Mo, Y. Chen and L. Jia, Appl. Energy, 2012, 93(5), 65-70. 30 L. Fedele, L. Colla and S. Bobbo, Int. J. Refrig., 2012, 35(5), 1359-1366.

31 H. Chen, Y. Ding and A. Lapkin, Powder Technol., 2009, 194(1-2), 132-141.

32 M. T. J. Zafarani, Chem. Thermodyn., 2012, 54, 55-67.

33 G. J. Lee, K. K. Chang, K. L. Min, et al., Thermochim. Acta, 2012, 542(542), 24-27.

34 V. S. Raykar and A. K. Singh, Thermochim. Acta, 2010, 502(1), 60-65.

35 E. V. Timofeeva, M. R. Moravek and D. Singh, J. Colloid Interface Sci., 2011, 364(1), 71-79.

36 K. Anoop, R. Sadr, J. Yu, et al., Int. Commun. Heat Mass Transfer, 2012, 39(9), 1325-1330.

37 C. Pang, J. Y. Jung, J. W. Lee, et al., Int. J. Heat Mass Transfer, 2012, 55(21-22), 5597-5602.

38 M. Saeedinia, M. A. Akhavan-Behabadi and M. Nasr, Exp. Therm. Fluid Sci., 2011, 36(1), 158-168.

39 S. M. Fotukian and M. N. Esfahany, Int. Commun. Heat Mass Transfer, 2010, 37(2), 214-219.

$40 \mathrm{~S}$. Harikrishnan and S. Kalaiselvam, Thermochim. Acta, 2012, 533(533), 46-55.

41 S. Soltani, S. G. Etemad and J. Thibault, Int. Commun. Heat Mass Transfer, 2010, 37(1), 29-33.

42 M. P. Beck, T. Sun and A. S. Teja, Fluid Phase Equilib., 2007, 260(2), 275-278.

43 J. Y. Jung, E. S. Kim and Y. T. Kang, Int. Commun. Heat Mass Transfer, 2012, 55(s7-8), 1941-1946.

44 M. R. Raveshi, A. Keshavarz, M. S. Mojarrad, et al., Exp. Therm. Fluid Sci., 2013, 44(1), 805-814.

45 W. Yu, H. Xie, L. Chen, et al., Colloids Surf., A, 2010, 355(1), 109-113.

46 L. S. Sundar, E. V. Ramana, M. K. Singh, et al., Chem. Phys. Lett., 2012, 554(6), 236-242.
47 H. Peng, G. Ding and H. Hu, Exp. Therm. Fluid Sci., 2011, 35(6), 960-970.

48 E. D. Robertis, E. H. H. Cosme, R. S. Neves, et al., Appl. Therm. Eng., 2012, 41(41), 10-17.

49 R. Kathiravan, R. Kumar, A. Gupta, et al., Int. Commun. Heat Mass Transfer, 2012, 33(2), 69-78.

50 Y. Xuan and L. I. J. Qiang, J. Eng. Thermophys., 2000, 21(1), 58-64.

51 T. X. Phuoc, Y. Soong and M. K. Chyu, Optic. Laser. Eng., 2007, 45(12), 1099-1106.

52 F. Su, X. Ma and Z. Lan, J. Taiwan Inst. Chem. Eng., 2011, 42(2), 252-257.

53 Z. H. Liu, X. F. Yang and J. G. Xiong, Int. J. Therm. Sci., 2010, 49(7), 1156-1164.

54 M. Zhaoguo, W. Daxiong, et al., Particuology, 2012, (5), 614618.

55 M. S. Liu, C. C. Lin, I. T. Huang, et al., Int. Commun. Heat Mass Transfer, 2005, 32(9), 1202-1210.

56 H. A. Mohammed, A. A. Al-Aswadi, N. H. Shuaib, et al., Renewable Sustainable Energy Rev., 2011, 15(6), 2921-2939.

57 H. Yu, S. Hermann, S. E. Schulz, et al., Chem. Phys., 2012, 408(408), 11-16.

58 X. Q. Wang and A. S. Mujumdar, J. Colloid Interface Sci., 2007, 46(1), 1-19.

59 S. A. Angayarkanni and J. Philip, Adv. Colloid Interface Sci., 2015, 225, 146-176.

60 I. Popa, G. Gillies, G. Papastavrou, et al., J. Phys. Chem. B, 2010, 114(9), 3170-3177.

61 W. Yu and H. Xie, J. Nanomater., 2012, 435873.

62 A. Bar-Hen, C. Bounioux and R. Yerushalmi-Rozen, J. Colloid Interface Sci., 2015, 452, 62-68.

63 L. Yang, K. Du, X. Niu, et al., Int. J. Refrig., 2011, 34(8), 17411748.

64 H. Yu, S. Hermann, S. E. Schulz, et al., J. Chem. Phys., 2012, 408(408), 11-16.

65 K. Gu, B. Chen and Y. Cheng, J. Rare Earths, 2013, 31(6), 589-594.

66 V. Zin, F. Agresti, S. Barison, et al., IEEE Trans. Nanotechnol., 2013, 12(5), 751-759.

67 G. Xia, H. Jiang, R. Liu, et al., Int. J. Therm. Sci., 2014, 84(4), 118-124.

68 Y. Gao, G. Chen, Y. Oli, et al., Mater. Sci. Eng., A, 2000, 252(5-6), 454-458.

69 H. K. Sang, R. C. Sun and D. J. Kim, J. Heat Transfer, 2007, 129(3), 298-307.

70 X. Li, D. Zhu and X. Wang, J. Colloid Interface Sci., 2007, 310(2), 456-463.

71 V. Kumaresan, Thermochim. Acta, 2012, 545, 180-186.

72 T. Yousefi, E. Shojaeizadeh, F. Veysi, et al., J. Sol. Energy, 2012, 86(2), 771-779.

73 A. Indhuja, K. S. Suganthi, S. Manikandan, et al., J. Taiwan Inst. Chem. Eng., 2013, 44(3), 474-479.

74 P. Garg, J. L. Alvarado and C. Marsh, Int. J. Heat Mass Transfer, 2009, 52(21-22), 5090-5101.

75 R. Wang, I. Bowlinga and W. Liub, RSC Adv., 2017, 7, 36763679. 
76 J. Kim, Y. S. Park, B. Veriansyah, et al., Chem. Mater., 2008, 20(20), 6301-6303.

77 N. Alzatecarvajal, E. V. Basiuk and V. Mezalaguna, RSC Adv., 2016, 6, 113596-113610.

78 S. S. Pati, V. Mahendran and J. Philip, J. Nanofluids, 2013, 2(2), 94-103.

79 H. Xie, H. Lee, W. Youn, et al., J. Appl. Phys., 2003, 94(8), 4967-4971.

80 S. S. J. Aravind, P. Baskar, T. T. Baby, et al., J. Phys. Chem. C, 2011, 115(34), 16737-16744.

81 T. P. Teng, H. G. Hsu, H. E. Mo, et al., J. Alloys Compd., 2010, 504(504), S380-S384.

82 H. K. Sang, R. C. Sun and D. J. Kim, J. Heat Transfer, 2007, 129(3), 298-307.

83 N. Kannadasan, K. Ramanathan and S. Suresh, Exp. Therm. Fluid Sci., 2012, 42(5), 64-70.

84 I. M. Mahbubul, I. M. Shahrul and S. S. Khaleduzzaman, Int. J. Heat Mass Transfer, 2015, 88, 73-81.

85 V. S. Nguyen, D. Rouxel and R. Hadji, Ultrason. Sonochem., 2011, 18(1), 382-388.

86 A. Ghadimi, R. Saidur and H. S. C. Metselaar, Int. J. Heat Mass Transfer, 2011, 54(17-18), 4051-4068.

87 H. J. Kim, I. C. Bang and J. Onoe, Optic. Laser. Eng., 2009, 47(5), 532-538.

88 X. Li, D. Zhu and X. J. Wang, J. Colloid Interface Sci., 2007, 310(2), 456-463.

89 B. V. Eremenko, T. N. Bezuglaya, A. N. Savitskaya, et al., Colloid J., 2001, 63(2), 173-178.

90 H. Zhu, C. Zhang, Y. Tang, et al., Carbon, 2007, 45(1), 226-228.

91 S. N. Himansu, RSC Adv., 2016, 6, 111889-111894.

92 X. Li, D. Zhu and X. Wang, J. Colloid Interface Sci., 2007, 310(2), 456-463.

93 X. Wei and W. Liqiu, Particuology, 2010, 8(3), 262-271.

94 S. A. Angayarkanni and J. Philip, J. Nanofluids, 2014, 3(1), 17-25.

95 D. Li, Y. Xie and H. Yong, RSC Adv., 2017, 7, 3727-3735.

96 C. Yang, X. Hou, Z. Li, et al., Appl. Surf. Sci., 2015, 388, 497502.
97 O. Elomaa, J. Oksanen and T. J. Hakala, Tribol. Int., 2014, 71(1), 62-68.

98 M. J. Kao and C. R. Lin, J. Alloys Compd., 2009, 483(1), 456459.

99 T. Luo, X. Wei, H. Zhao, et al., Ceram. Int., 2014, 40(7), 10103-10109.

100 M. Khadem, O. V. Penkov and V. E. Pukha, RSC Adv., 2016, 6(62), 56918-56929.

101 L. Wu, Y. Zhang and G. Yang, $R S C A d v ., 2016$, 6, 6983669844.

102 C. Zhang, S. Zhang, L. Yu, et al., Appl. Surf. Sci., 2012, 259(42), 824-830.

103 M. D. Abad and J. C. Sánchez-López, Wear, 2013, 297(1-2), 943-951.

104 B. Chen, K. Gu, J. Fang, et al., Appl. Surf. Sci., 2015, 353, 326-332.

105 R. Chou, A. H. Battez and J. J. Cabello, Tribol. Int., 2010, 43(12), 2327-2332.

106 T. Sui, B. Song and F. Zhang, RSC Adv., 2016, 6(1), 393-402. 107 L. Kong, J. Sun and Y. Bao, Wear, 2017, WEA101998.

108 X. L. Wang, Y. L. Yin, G. N. Zhang, et al., Phys. Procedia, 2013, 50, 466-472.

109 J. Ma, Y. Mo and B. Mingwu, Wear, 2009, 266(7-8), 627-631.

110 M. Flores-Castañeda, E. Camps, M. Camacho-López, et al., J. Alloys Compd., 2014, 643, S67-S70.

111 C. C. Chou and S. H. Lee, J. Mater. Process. Technol., 2008, 201(1), 542-547.

112 B. Zareh-Desari and B. Davoodi, J. Cleaner Prod., 2016, 135, 1198-1209.

113 X. Zheng, Y. Xu, J. Geng, et al., Tribol. Int., 2016, 102, 79-87.

114 X. F. Sun, Y. L. Qiao, W. Song, et al., Phys. Procedia, 2013, 50, 343-347.

115 P. J. Shi, H. L. Yu, H. M. Wang, et al., Phys. Procedia, 2013, 50, 461-465.

116 Y. Choi, C. Lee, Y. Hwang, et al., Curr. Appl. Phys., 2009, 9(2), e124-e127. 